\title{
The new hybrid BBN model with the photon cooling, $X$ particle, and the primordial magnetic field
}

\author{
Dai G. Yamazaki* ${ }^{*}, \|$, Motohiko Kusakabe ${ }^{\ddagger}$, Toshitaka Kajino ${ }^{\dagger, \S}$, \\ Grant J. Mathews ${ }^{\ddagger}$ and Myung-Ki Cheoun $\llbracket$ \\ *University Education Center, Ibaraki University, \\ 2-1-1 Bunkyo, Mito, Ibaraki 310-8512, Japan \\ ${ }^{\dagger}$ National Astronomical Observatory of Japan, \\ 2-21-1 Osawa, Mitaka, Tokyo 181-8588, Japan \\ ${ }^{\ddagger}$ Center for Astrophysics, \\ Department of Physics, University of Notre Dame, \\ Notre Dame, IN 46556, USA \\ $\S$ Department of Astronomy, Graduate School of Science, \\ The University of Tokyo, 7-3-1 Hongo, \\ Bunkyo-ku, Tokyo 113-0033, Japan \\ IDepartment of Physics, Soongsil University, \\ Seoul 156-743, Korea \\ "yamazaki.dai@nao.ac.jp \\ Received 5 October 2015 \\ Revised 4 March 2016 \\ Accepted 8 June 2017 \\ Published 24 August 2017
}

\begin{abstract}
The Big Bang Nucleosynthesis theory accurately reproduces the abundances of light elements in the universes, except for the ${ }^{7} \mathrm{Li}$ abundance. The calculated ${ }^{7} \mathrm{Li}$ abundance with the baryon-to-photon ratio fixed by the observations of the cosmic microwave background $(\mathrm{CMB})$ is inconsistent with the observed lithium abundances on the surface of metal-poor halo stars, and this problem is called "7 $\mathrm{Li}$ problem". Previous studies proposed to resolve this ${ }^{7} \mathrm{Li}$ problem include photon cooling (possibly via the Bose-Einstein condensation of a scalar particle), the decay of a long-lived $X$ particle (possibly the nextto-lightest supersymmetric particle), or an energy density of a primordial magnetic field (PMF). We review and analyze the results of these solutions both separately and in concert, and the constraint on the $X$ particles and the PMF parameters from observed light-element abundances with a likelihood analysis. We can discover parameter ranges of the $X$ particles which can solve the ${ }^{7} \mathrm{Li}$ problem and constrain the energy density of the PMF.
\end{abstract}

Keywords: Big Bang Nucleosynthesis; dark matter; magnetic field.

PACS Number(s): 26.35.+c, 98.80.Ft

${ }$ Corresponding author.

This is an Open Access article published by World Scientific Publishing Company. It is distributed under the terms of the Creative Commons Attribution 4.0 (CC-BY) License. Further distribution of this work is permitted, provided the original work is properly cited. 


\section{Introduction}

Physical processes in the early universe are probed with several important astronomical observations. Among the observations, primordial abundances of light elements provide information of the Big Bang Nucleosynthesis (BBN), and the cosmic microwave background (CMB) and the matter power spectrum (MPS) provide information of the evolution of the primordial perturbations as well as the formation and evolution of the primordial objects after the last scattering of the CMB photons.

The baryon-to-photon ratio, $\eta$, is one of the important parameters characterizing the BBN model. Recently, we have constrained $\eta$ from observations of $\mathrm{CMB}^{15}$ and tested various models of BBN using the constrained value of $\eta$ as an input.

The BBN theory can accurately reproduce the abundances of light elements except for ${ }^{7} \mathrm{Li}$ with $\eta$ fixed by the CMB observations ${ }^{1 / 5}$ However the primordial abundance of ${ }^{7} \mathrm{Li}$ from this BBN theory differs from ${ }^{7} \mathrm{Li}$ abundances on the surface of metal-poor halo stars inferred from astronomical observations. ${ }^{[6]}$ It is called the "Li problem." [7 To search for an optimum theoretical model for this dilemma, we discuss three possible effects on BBN and show a resolution to the Li problem in this paper.

A first candidate solution to the ${ }^{7} \mathrm{Li}$ problem is photon cooling. If the photons are cooled after the end of $\mathrm{BBN}, \eta$ during $\mathrm{BBN}$ is smaller than the present value and the overproduction of ${ }^{7} \mathrm{Li}$ can be prevented.

If a scalar particle forms a Bose-Einstein condensate (BEC) during the postBBN epoch, but before the recombination, such a particle can cool the photons. Although Refs. 8 and 9 indicated that the dark matter axion was a candidate for this BEC, as discussed in Ref. 33, this cooling from the BEC particle occurs too late to agree with the chemical potential $\mu$ and the Compton $y$ parameter constrained by the CMB observations. Reference 10, however, illustrates that the photons can also be cooled by the resonant oscillations between light Abelian gauge bosons in the hidden sector and photons as another possibility.

The radiative decay of a hypothetical exotic long-lived $(X)$ particle after the end of $\mathrm{BBN}^{111}$ is, also, one of the innovative solutions to the ${ }^{7} \mathrm{Li}$ problem. Since the deuterium is overproduced in the photon-cooling model, it is impossible to resolve the ${ }^{7} \mathrm{Li}$ problem by the cooling alone $\frac{12[13}{}$ On the other hand, if we simultaneously assume the photon cooling and the decay particle model, the problem of deuterium overproduction is solved ${ }^{11}$ This hybrid model which consists of a radiatively decaying particle and photon cooling decreases the primordial D abundance through the photodisintegration reaction ${ }^{2} \mathrm{H}(\gamma, n)^{1} \mathrm{H}$, by nonthermal photons generated by the decaying $X$ particles. $12-30$

As a third possibility we consider the energy density of a primordial magnetic field (PMF), $\rho_{\mathrm{MF}}$. Since $\rho_{\mathrm{MF}}$ can boost the cosmic expansion rate and the results of the BBN model with a PMF differ from those without a PMF, 31 the solution to the Li problem can be improved by a PMF. 
We introduce the combined effects of photon cooling, the radiative decay of an $X$ particle, and a PMF on BBN, and derive a reasonable solution to Li problem in this paper. We, also, show constraints on the $X$ particles and the PMF parameters by observed light-element abundances with a likelihood analysis, and discuss the effective number of relativistic degrees of freedom with our results.

\section{Model}

In this section, we introduce the new hybrid BBN model with the effects of (i) photon cooling, (ii) the radiative decay of the $X$-particle, and (iii) the energy density of a PMF. We have made an assumption that the photon cooling occurs after both BBN and $X$ decay. In this study, we use the modified Kawano BBN code ${ }^{34[35}$ considering this new hybrid BBN model.

The estimation method of the $X$ decay effect on BBN is similar to that described in Ref. 11. Important effects of a PMF on BBN were studied in Ref. 31, and results of BBN with a PMF were shown as a function of $\eta$ and the parameters of a PMF were constrained in Ref. 33 . The reaction rates are taken from the JINA REACLIB Database V1.0 $\stackrel{36}{[}$ A neutron lifetime is given by $\tau_{n}=878.5\left( \pm 0.7_{\text {st }} \pm 0.3_{\text {sy }}\right)$ s. $\stackrel{\text { [7] }}{\text { As }}$ for nonthermal nucleosynthesis, we have used updated reaction rates of ${ }^{4} \mathrm{He}$ photodisintegration ${ }^{28}$ derived from the cross-section data using precise measurements with laser-Compton photons. $\underline{3839}$

Since the baryon-to-photon ratio $\eta$ affects transfer functions of nonthermal nuclides, we should correct it for changes in both the nuclear abundances and the $\eta$. In this study, we update the transfer functions by a self-consistent BBN calculation as a function of $\eta$. We also update the energy loss rate for nonthermal photons, and the rates for energy loss and the destruction of nonthermal nuclei produced by the energetic photons. Nuclear transfer functions are calculated for 11 different $\eta$ values in this paper. We then interpolate the transfer functions as a function of $\eta$ to derive calculated light-element abundances.

We then compare the results of light-element abundances from the BBN model to observational abundances that are summarized in the second column of Table 1

Table 1. Agreement with observed light-element abundances for the five models considered here.

\begin{tabular}{|c|c|c|c|c|c|c|}
\hline \multicolumn{2}{|c|}{ Model } & SBBN & $\gamma$-Cooling & $X$ & PMF & $\gamma$-Cooling $+X+\mathrm{PMF}$ \\
\hline \multicolumn{2}{|c|}{$\eta$} & $\eta_{0}^{[\mathrm{a}]}$ & $\eta_{\mathrm{BBN}}^{[\mathrm{b}]}$ & \multicolumn{2}{|c|}{$\eta_{0}^{\lfloor\mathrm{a}\rfloor}$} & $\eta_{\mathrm{BBN}}^{[\mathrm{b}]}$ \\
\hline Nuclide & Observation & & & & & \\
\hline$\overline{Y_{p}\left({ }^{4} \mathrm{He}\right)}$ & $0.2345-0.2777^{[\mathrm{c}]}$ & $\checkmark$ & $\checkmark$ & $\checkmark$ & $\checkmark$ & $\checkmark$ \\
\hline $\mathrm{D} / \mathrm{H}\left(\times 10^{5}\right)$ & $2.37-2.85$ & $\checkmark$ & - & $\checkmark$ & $\checkmark$ & $\checkmark$ \\
\hline${ }^{3} \mathrm{He} / \mathrm{H}\left(\times 10^{5}\right)$ & $0-3.1$ & $\checkmark$ & $\checkmark$ & $\checkmark$ & $\checkmark$ & $\checkmark$ \\
\hline${ }^{7} \mathrm{Li} / \mathrm{H}\left(\times 10^{10}\right)$ & $1.06-2.35^{[\mathrm{d}]}$ & - & $?$ & - & - & $\checkmark$ \\
\hline${ }^{6} \mathrm{Li} / \mathrm{H}\left(\times 10^{12}\right)$ & $0-9.5^{[\mathrm{e}]}$ & $\checkmark$ & $\checkmark$ & $\checkmark$ (high) & $\checkmark$ & $\checkmark$ (high) \\
\hline
\end{tabular}

Note: ${ }^{\mathrm{a}} \eta_{0}=(6.19 \pm 0.14) \times 10^{-10,9,62 \mathrm{~b}} \eta_{\mathrm{BBN}}=(2 / 3)^{3 / 4} \eta_{0} \cdot{ }^{c}$ Conservative limit $63 \mathrm{~d}$ Spite plateau value 6 in metal-poor halo stars. ${ }^{\text {e The }} 2 \sigma$ upper limit from a spectral analysis of metal-poor halo stars 64 
(see Ref. 32 for details). We use constraints on $\eta$ as derived from the WMAP 9-year analysis,

$$
\eta=\frac{n_{b}}{n_{\gamma}}=(6.19 \pm 0.14) \times 10^{-10}
$$

where $n_{b}$ and $n_{\gamma}$ are the number densities of baryons and photons, respectively. In this study, the number of neutrino species is $N_{\nu}=3$, and it is not to be confused with the effective number of neutrino species $N_{\text {eff }}$. We will discuss it below. From the WMAP 9-year analysis, $\frac{5}{5}$ the degeneracy between $N_{\text {eff }}$ and $\eta$ can be negligible as shown in Ref. 33 .

\subsection{Photon cooling}

If the photon cooling occurs after both BBN and $X$ decay, the photon number density at the BBN is greater than that without the photon cooling. Thus, the baryon-to-photon ratio at the $\mathrm{BBN}$ epoch, $\eta_{\mathrm{BBN}}$, will be less than the value constrained by the CMB observations. Considering the photon cooling, the predicted baryon-to-photon ratio by the BEC mode ${ }^{9}$ is smaller by a factor of $(2 / 3)^{3 / 4}$ at the BBN epoch: $\eta_{\mathrm{BBN}}=(4.57 \pm 0.10) \times 10^{-10}$. In this model, the abundances of $\mathrm{D},{ }^{3} \mathrm{He}$, and ${ }^{6} \mathrm{Li}$ increase, while those of ${ }^{4} \mathrm{He}$ and ${ }^{7} \mathrm{Li}$ decrease. This model can reproduce the abundance of ${ }^{7} \mathrm{Li}$ consistent with the observed one, while it leads to an undesirable consequence in the D abundance.

\section{2. $X$ particle model}

Nonthermal photons can be produced by nonthermal electromagnetic energy injection from the radiative decay of a long-lived massive $X$ particle in the postBBN epoch. 14|15 19:20 Particle theories beyond the standard model ${ }^{40 \mid 41}$ predict such decaying particles. At first, the $X$ particles decay radiatively and produce energetic photons, and then, these photons react with background photons and this reaction creates $e^{+} e^{-}$pairs. Finally, an electromagnetic cascade shower develops. Since an upper cutoff of the spectrum of nonthermal photons is given by $E_{C}=m_{e}^{2} / 22 T$, where $m_{e}$ is the electron mass, $\frac{20}{2}$ the upper cutoff energies become relatively smaller at higher temperature in the early universe.

The nonthermal photons produced by the particle decay gradually disintegrate the light nuclei produced in BBN with decreasing temperature of the universe. 13 15/2324]27 Since the threshold energies for ${ }^{7} \mathrm{Be}$ and D photodisintegration are relatively small as follows: $1.5866 \mathrm{MeV}$ and $2.2246 \mathrm{MeV}$, respectively, they are most strongly photodisintegrated. ${ }^{7} \mathrm{Be}$ and $\mathrm{D}$ are mainly photodisintegrated via the reactions ${ }^{7} \mathrm{Be}+\gamma \rightarrow{ }^{3} \mathrm{He}+{ }^{4} \mathrm{He}$ and $\mathrm{D}+\gamma \rightarrow n+p$, where $\gamma \mathrm{s}$ represent nonthermal photons. Disintegrations of ${ }^{7} \mathrm{Be}$ and $\mathrm{D}$ start at an early time $\left(t \lesssim 10^{6} \mathrm{~s}\right)$ which corresponds to $T \gtrsim 10^{7} \mathrm{~K}$ when the energies of cutoff exceed the threshold energies. Thus, in case that the cutoff energy is more than the threshold energies for the destructions of ${ }^{7} \mathrm{Be}$ and $\mathrm{D}$, but less than those of other nuclei, ${ }^{7} \mathrm{Be}$ and $\mathrm{D}$ nuclei are destroyed by the nonthermal photon field while other nuclei survive. 
Since, long time after the end of the BBN epoch, ${ }^{7} \mathrm{Be}$ nuclei are converted to ${ }^{7} \mathrm{Li}$ nuclei by electron capture as follows: ${ }^{7} \mathrm{Be}+e^{-} \rightarrow{ }^{7} \mathrm{Li}+\nu_{e}$, the primordial abundances of ${ }^{7} \mathrm{Li}$ correspond to the sum of the abundances of ${ }^{7} \mathrm{Li}$ and ${ }^{7} \mathrm{Be}$ at the end of the BBN epoch. Therefore, the reduction of the primordial ${ }^{7} \mathrm{Li}$ abundance is realized by the photodisintegration of ${ }^{7} \mathrm{Be}$ via decaying $X$ particles. As shown in Table 1 the photon-cooling model has a problem of D overproduction. If we simultaneously assume the photon cooling and the decay particle model, this problem can be resolved.

To research quantitative effects of the decaying $X$ particle on BBN, we characterize the effects of the electromagnetic energy injection by two parameters. One is defined by $\zeta_{X}=\left(n_{X}^{0} / n_{\gamma}^{0}\right) E_{\gamma 0}$. Here, $\left(n_{X}^{0} / n_{\gamma}^{0}\right)$ is the ratio of the number density of decaying $X$ particles to the number density of the background photons before the decay of the $X$ particles and $E_{\gamma 0}$ is the energy of the photon emitted by the radiative decay. The other parameter $\tau_{X}$ indicates the decay lifetime of the $X$ particle $[1]$

\subsection{PMF model}

A PMF affects many physical phenomena in the early universe. The CMB is affected by the PMF 424 The peak position and the amplitude of the MPS is also changed by a PMF ${ }^{45146}$ We can constrain the PMF parameters from the CMB and MPS observations. $\stackrel{4754}{54}$ When we consider the energy density of a PMF in the BBN epoch, the rate of the comic expansion is more rapid than without a PMF. Therefore, we take into account the effect of the PMF in BBN. Since the freeze-out of the weak reactions occur earlier, the neutron abundance at the freeze-out increases. The neutrons are decaying after the freeze-out, and the interval between the freeze-out and ${ }^{4} \mathrm{He}$ production also becomes shorter by the faster cosmic expansion. Therefore, more neutrons survive at the start of the ${ }^{4} \mathrm{He}$ production epoch. As a result, $\frac{31 \mid 32}{\sqrt{3}}$ the ${ }^{4} \mathrm{He}$ abundance increases significantly, on the other hand, the D and ${ }^{3} \mathrm{He}$ abundances increase moderately, the ${ }^{6} \mathrm{Li}$ abundance increases slightly, and the ${ }^{7} \mathrm{Li}$ abundance decreases.

\subsection{Likelihood analysis}

By using a likelihood analysis, we constrain the parameters of the $X$ particle and the PMF. The likelihood function for the observed value $A_{\text {obs }}$ can be expressed as

$$
L_{A}\left(\tau_{X}, \zeta_{X}, \rho_{B}\right)=\frac{1}{\sqrt{2 \pi} \sigma_{A_{\mathrm{obs}}}} \exp \left[\frac{-\left(A_{\mathrm{th}}\left(\tau_{X}, \zeta_{X}, \rho_{B}\right)-A_{\mathrm{obs}}\right)^{2}}{2 \sigma_{A_{\mathrm{obs}}}^{2}}\right],
$$

where $A_{\mathrm{th}}\left(\tau_{X}, \zeta_{X}, \rho_{B}\right)$ is from the theoretical calculation for each $\eta$ value by our code and values of $A_{\text {obs }}$ are shown in Table 1. For constraint on $\left(\tau_{X}, \zeta_{X}, \rho_{B}\right)$, we utilize the combined likelihood functions as follows:

$$
L_{\mathrm{LE}}\left(\tau_{X}, \zeta_{X}, \rho_{B}\right)=L_{\mathrm{D}} \times L_{3} \mathrm{He} \times L_{4} \mathrm{He} \times L_{6}{ }_{\mathrm{Li}} \times L_{7} \mathrm{Li} .
$$


D. G. Yamazaki et al.

Here, the likelihood functions for respective parameters are defined by

$$
\begin{aligned}
L_{\mathrm{LE}}\left(\tau_{X}\right) & =\iint d \zeta_{X} d \rho_{B} L_{\mathrm{LE}}\left(\tau_{X}, \zeta_{X}, \rho_{B}\right), \\
L_{\mathrm{LE}}\left(\zeta_{X}\right) & =\iint d \tau_{X} d \rho_{B} L_{\mathrm{LE}}\left(\tau_{X}, \zeta_{X}, \rho_{B}\right), \\
L_{\mathrm{LE}}\left(\rho_{B}\right) & =\iint d \zeta_{X} d \tau_{X} L_{\mathrm{LE}}\left(\tau_{X}, \zeta_{X}, \rho_{B}\right) .
\end{aligned}
$$

\section{Results and Discussions}

In this section, we show the results of constraint on the $X$ particle and the PMF parameters in the new hybrid BBN models from a maximum likelihood analysis. We derive the constraint on $\tau_{X}, \zeta_{X}$, and $B^{2} \propto \rho_{B}$ as listed in Table 2 where $B$ is the comoving and scale-invariant strength of the PMF $32 \sqrt[44]{4}$ The probability distributions of $\tau_{X}, \zeta_{X}$, and $B^{2} \propto \rho_{B}$ are also given by Fig. 1 The blue curves in Fig. 1 indicate the probability distributions. The limited values of $\zeta_{X}$ and $\tau_{X}$ are given by

$$
\begin{array}{r}
4.06<\log _{10}\left(\tau_{X}[\mathrm{~s}]\right)<6.10 \quad(2 \sigma, 95 \% \text { C.L. }) \\
-9.70<\log _{10}\left(\zeta_{X}[\mathrm{GeV}]\right)<-6.23 \quad(2 \sigma, 95 \% \text { C.L. }) .
\end{array}
$$

In this study, the BBN model with a PMF gives a better likelihood than without a PMF, and the best fit of the energy density of a PMF is given by

$$
\rho_{\mathrm{PMF}}(a)=\frac{\rho_{\mathrm{PMF}}\left(a_{0}\right)}{a^{4}}=6.82 \times 10^{-52} a^{-4} \mathrm{GeV}^{4} \quad \text { (the best fit). }
$$

Here $a$ is the scale factor and $a_{0}$ is the value at the present time. This best fit value corresponds to

$$
B(a)=1.89 a^{-2} \mu \mathrm{G} \quad(\text { the best fit }),
$$

where we use $\rho_{B}=B^{2} / 8 \pi=1.9084 \times 10^{-40} \times(B / G)^{2} \mathrm{GeV}^{4}$. An upper bound on the PMF energy density at the $2 \sigma$ confidence level is also obtained,

$$
\rho_{\mathrm{PMF}}(a)=\frac{\rho_{\mathrm{PMF}}\left(a_{0}\right)}{a^{4}}<1.45 \times 10^{-51} a^{-4} \mathrm{GeV}^{4} \quad(2 \sigma, 95 \% \text { C.L. }) .
$$

This upper bound corresponds to

$$
B(a)<3.05 a^{-2} \mu \mathrm{G} \quad(2 \sigma, 95 \% \text { C.L. }) .
$$

Table 2. Constraints on parameters of the $X$ particle and the PMF amplitude.

\begin{tabular}{lr}
\hline Constrained values $(95 \%$ C.L. $)$ & Best \\
\hline $4.06<\log _{10}\left(\tau_{X}[\mathrm{~s}]\right)<6.10$ & 4.34 \\
$-9.70<\log _{10}\left(\zeta_{X}[\mathrm{GeV}]\right)<-6.23$ & -9.55 \\
$B<3.05 \mu \mathrm{G}$ & $1.89 \mu \mathrm{G}$ \\
Confidence intervals $(2 \sigma, 95 \%$ C.L. $)$ and upper bounds $(2 \sigma, 95 \%$ C.L. $)$ & \\
$\quad$ on strengths of PMFs.
\end{tabular}



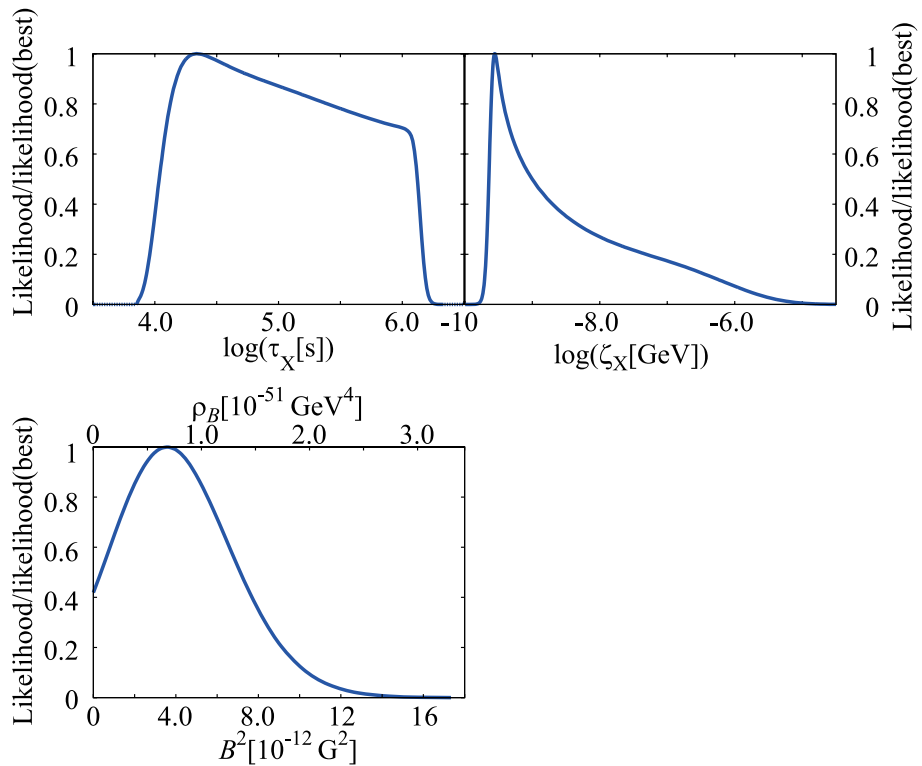

Fig. 1. Likelihood distributions for parameters of the $X$ particle and a PMF. The upper-left, upper-right, and bottom-left panels show distributions of $\tau_{X}, \zeta_{X}$, and $B^{2} \propto \rho_{B}$, respectively. The vertical axes in the all panels are normalized to their maximum likelihood values.

Source: This figure is reproduced from Ref. 33.

Figure 2 indicates contours of $1 \sigma$ and $2 \sigma$ confidence limits on various planes of the $X$ particle and PMF parameters (i.e., $\tau_{X}$ versus $\zeta_{X}, \rho_{B}$ versus $\zeta_{X}$, and $\rho_{B}$ versus $\left.\tau_{X}\right)$. The black and gray (color version: blue and aqua) contours show the $1 \sigma$ $(68 \%)$ and $2 \sigma(95 \%)$ confidence limits. These parameters are strongly degenerate.

Figure 3 shows an allowed region in the $\left(\tau_{X}, \zeta_{X}\right)$-plane by the observational constraints on the light-element abundances for $\eta=4.57 \times 10^{-10}$ and $B=1.89 \mu \mathrm{G}$. The abundance of ${ }^{4} \mathrm{He}$ does not constrain the $X$ particle parameters $\left(\tau_{X}, \zeta_{X}\right)$ strongly, while the energy density of a PMF is mainly limited by the abundance of ${ }^{4} \mathrm{He}$. Figure 2 shows that there is not a degeneracy between the parameters of the $X$ particle and the PMF in the allowed region.

It is worthwhile to make a comparison between the constraints on parameters from the observed light-element abundances in the context of the hybrid model described here and constraints based only upon the observed CMB and ${ }^{4} \mathrm{He}$ abundance. Considering first a comparison between the total radiative energy densities derived from the BBN and the $\mathrm{CMB}$. The total radiative energy density in a model with photon cooling and a PMF is given by

$$
\begin{aligned}
\rho_{R} & =\rho_{\mathrm{CMB}}+\rho_{\nu}+\rho_{s}+\rho_{\mathrm{PMF}} \\
& =\rho_{\mathrm{CMB}}\left[1+\left(N_{\nu}+0.046\right) D \frac{3}{2}+\frac{1}{2}+\frac{\rho_{\mathrm{PMF}}}{\rho_{\mathrm{CMB}}}\right]
\end{aligned}
$$




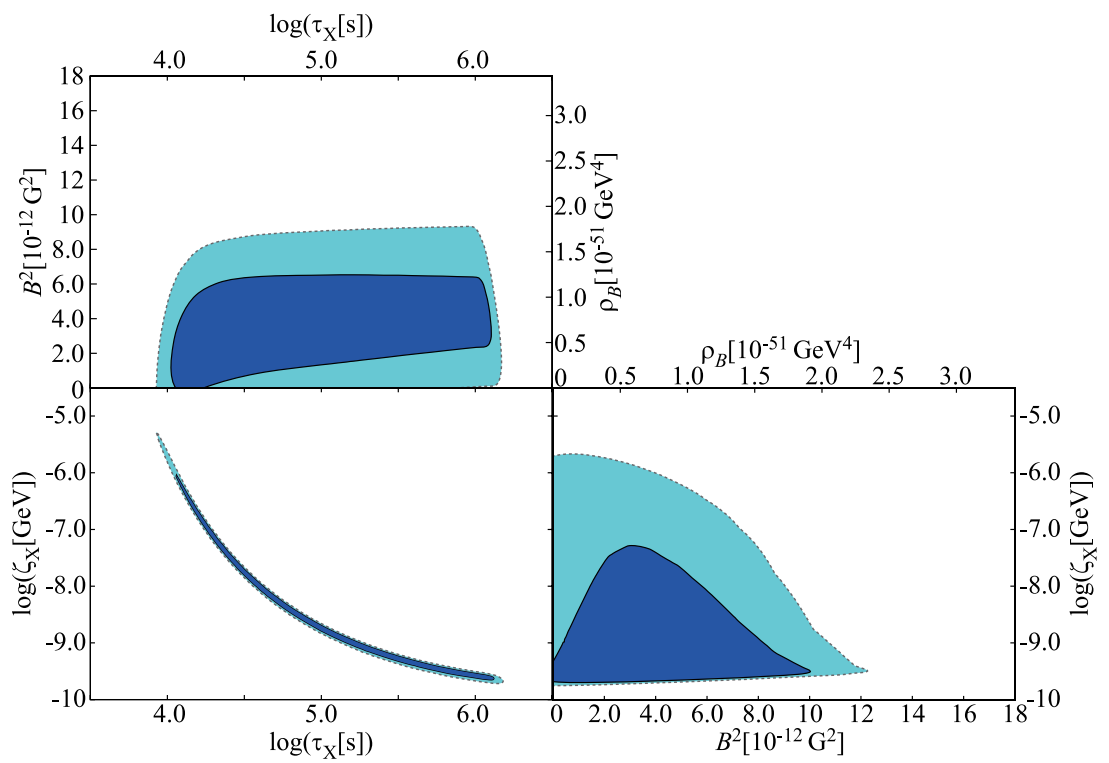

Fig. 2. (Color online) Contours of $1 \sigma$ and $2 \sigma$ confidence limits. The upper-left, bottom-left, and bottom-right panels show parameter planes of $\rho_{B}$ versus $\tau_{X}, \tau_{X}$ versus $\zeta_{X}$, and $\rho_{B}$ versus $\zeta_{X}$, respectively. The black and gray (color version: blue and aqua) contours show the $1 \sigma(68 \%)$ and $2 \sigma(95 \%)$ confidence limits.

Source: This figure is reproduced from Ref. 33]

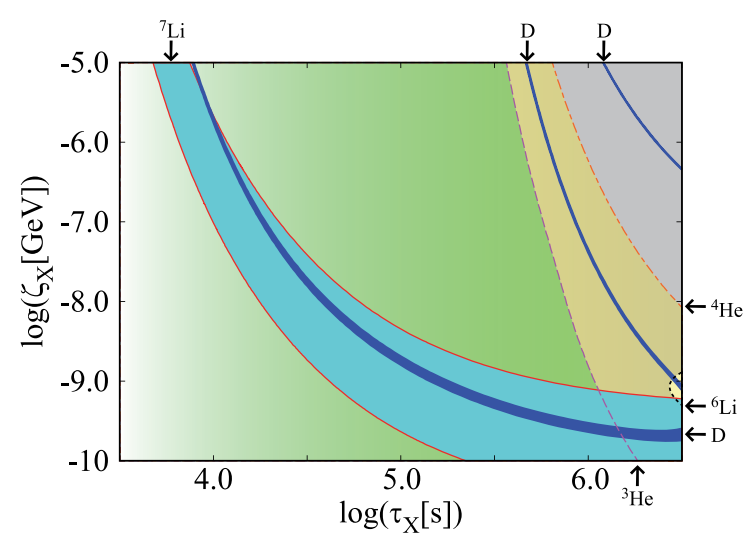

Fig. 3. (Color online) Allowed regions in the $\left(\tau_{X}, \zeta_{X}\right)$-plane by the observational constraints on the light-element abundances for $\eta=4.57 \times 10^{-10}$ and $B=1.89 \mu \mathrm{G}$. The curves denote the allowed regions derived from observational limits on the primordial elemental abundances. The narrow dark band and the region bounded by the solid curves (color version: blue and aqua regions) show the $2 \sigma(95 \%)$ confidence limits determined from the observed abundances of $\mathrm{D}$ and ${ }^{7} \mathrm{Li}$, respectively. Dashed, dot-dashed, and dotted curves (color version: purple, orange, and black curves) are the $2 \sigma(95 \%)$ confidence limits determined from the upper limits on the ${ }^{3} \mathrm{He},{ }^{4} \mathrm{He}$, and ${ }^{6} \mathrm{Li}$ abundances.

Source: This figure is reproduced from Ref. 33 


$$
\begin{aligned}
& =\rho_{\mathrm{CMB}}\left\{1+\left[\frac{3}{2}\left(N_{\nu}+0.046\right)+\frac{1}{2 D}+\frac{\rho_{\mathrm{PMF}}}{D \rho_{\mathrm{CMB}}}\right] D\right\} \\
& =\rho_{\mathrm{CMB}}\left(1+N_{\mathrm{eff}} D\right),
\end{aligned}
$$

where $\rho_{\mathrm{CMB}}, \rho_{\nu}$, and $\rho_{s}$ are the energy densities of the CMB photons, neutrinos, and excited scalar particles, respectively, after the photon cooling, $N_{\nu}$ is the number of neutrino species, and the number of 0.046 in these equations is derived from a slight heating of the three neutrinos species during $e^{+} e^{-}$annihilation. We define $D$ and $N_{\text {eff }}$ in Eq. (13) as follows:

$$
\begin{aligned}
D & =\frac{7}{8}\left(\frac{4}{11}\right)^{\frac{4}{3}} \simeq 0.227 \\
N_{\mathrm{eff}} & =\left[\frac{3}{2}\left(N_{\nu}+0.046\right)+\frac{1}{2 D}+\frac{\rho_{\mathrm{PMF}}}{D \rho_{\mathrm{CMB}}}\right] .
\end{aligned}
$$

From the upper limit on PMF energy density of Eqs. (11) and (15), the $2 \sigma$ upper limit to $N_{\text {eff }}$ in our hybrid model with a PMF becomes

$$
N_{\text {eff }}<8.27 \text {. }
$$

From the WMAP 9-year data, on the other hand, only a lower limit of $N_{\text {eff }}>$ $1.7(2 \sigma)$ is obtained. Using CMB data of WMAP together with other measurements of power spectrum at higher multipoles $\ell$ (SPT, $\stackrel{55[56}{\mathrm{ACT}^{57 / 58}}$ ) and Supernova data (SNLS3 39 ), however, $N_{\text {eff }}$ is better constrained to be $N_{\text {eff }}=3.66_{-0.39}^{+0.40}$. We note that constraints on $N_{\text {eff }}$ from these observed datasets generally do not consider the

\begin{tabular}{|c|c|c|c|c|}
\hline \multicolumn{5}{|c|}{ Fixed $N_{\text {eff }}=3.046$ with WMAP only } \\
\hline & WMAP & $\mathrm{W}+\mathrm{H} 0$ & $\mathrm{~W}+\mathrm{Bao}$ & $\mathrm{W}+\mathrm{H} 0+\mathrm{Bao}$ \\
\hline$\eta$ & $6.19 \pm 0.14$ & $6.26 \pm 0.13$ & $6.15 \pm 0.12$ & $6.20 \pm 0.12$ \\
\hline \multicolumn{5}{|c|}{ Free $N_{\text {eff }}$ with WMAP only } \\
\hline$N_{\text {eff }}$ & $>1.7(2 \sigma)$ & $3.96_{-0.74}^{+0.75}$ & $4.9_{-2.2}^{+2.4}$ & $4.23 \pm 0.59$ \\
\hline$\eta$ & $6.20 \pm 0.14$ & $6.20 \pm 0.13$ & $6.16 \pm 0.12$ & $6.16 \pm 0.12$ \\
\hline \multicolumn{5}{|c|}{ Fixed $N_{\text {eff }}=3.046$ with WMAP + ACT + SPT + SNLS3 } \\
\hline & WMAP & $\mathrm{W}+\mathrm{H} 0$ & $\mathrm{~W}+\mathrm{Bao}$ & $\mathrm{W}+\mathrm{H} 0+\mathrm{Bao}$ \\
\hline$\eta$ & $6.12 \pm 0.10$ & $6.146_{-0.096}^{+0.097}$ & $6.055_{-0.090}^{+0.091}$ & $6.085 \pm 0.09$ \\
\hline \multicolumn{5}{|c|}{ Free $N_{\text {eff }}$ with WMAP $+\mathrm{ACT}+\mathrm{SPT}+\mathrm{SNLS} 3$} \\
\hline$N_{\text {eff }}$ & $3.97 \pm 0.66$ & $3.66_{-0.39}^{+0.40}$ & $3.61 \pm 0.60$ & $3.83 \pm 0.40$ \\
\hline$\eta$ & $6.23 \pm 0.13$ & $6.186 \pm 0.099$ & $6.12 \pm 0.11$ & $6.148_{-0.093}^{+0.097}$ \\
\hline
\end{tabular}
consistency with the light-element abundances except for ${ }^{4} \mathrm{He}$. If the hybrid model of photon cooling and $X$ decay is the true solution, a theoretical estimation of $N_{\text {eff }}$ using parameter values constrained from observational light-element abundances

Table 3. Constraints on $N_{\text {eff }}$ and $\eta$ from the CMB. 
can provide one important constraint on $N_{\text {eff }}$ independent of the observed CMB data (Table 3). If we adopt the $2 \sigma$ upper limit of $N_{\text {eff }}<4.06$ from the combined $\mathrm{WMAP}+\mathrm{SPT}+\mathrm{ACT}+\mathrm{SNLS} 3$ dataset, the present model is excluded even if the amplitude of the PMF is zero. It is, however, possible that photon cooling is less efficient than that assumed in this study, or that there is an energy transfer from the PMF to the CMB via dissipation after BBN. In both cases, the resulting $N_{\text {eff }}$ values are smaller than the present result, and the parameter regions of the $X$ particle and the PMF would change accordingly. The constraint on $N_{\text {eff }}$ from CMB observations can then be used to limit the combined effects of photon cooling, $X$ decay, and the PMF.

\section{Summary}

We have calculated BBN taking into account the three possible effects of photon cooling, the radiative decay of $X$ particles, and a PMF simultaneously, and extended the standard BBN to this new hybrid BBN. We then used a maximum likelihood analysis to constrain the parameters of the $X$ particles and the energy density of the PMF by the observed abundances of light elements up to Li. As a result, we obtained allowed ranges for the $X$ particle parameters and find that the new hybrid model with a PMF gives the better likelihood than that without a PMF.

We discussed the degeneracy between the parameters of the $X$ particle and the PMF. Since the $X$ particle parameters are mainly limited by the $\mathrm{D}$ and ${ }^{7} \mathrm{Li}$ abundances, while the PMF energy density is mainly limited by the ${ }^{4} \mathrm{He}$ abundance, we found that the parameters of the PMF and the $X$ particle have no significant degeneracies (Fig. 2).

We also discussed the effective number of neutrino species $N_{\text {eff }}$ with our new hybrid model. Since the constraint on $N_{\text {eff }}$ from the CMB observations is different from the $N_{\text {eff }}$ value in our hybrid model which is consistent with the observed light elements, it is difficult to directly compare these two $N_{\text {eff }}$ values. We will report a new limit on $N_{\text {eff }}$ derived by taking into account analyses of both BBN and the CMB simultaneously in our future work.

\section{Acknowledgments}

This work was supported in part by Grants-in-Aid for Scientific Research of JSPS (Dai G. Yamazaki by Grant No. 25871055, Toshitaka Kajino by Grant Nos. 20105004 and 24340060). Work at the University of Notre Dame (Grant J. Mathews) is supported by the U.S. Department of Energy under Nuclear Theory Grant No. DE-FG02-95-ER40934. Work (Myung-Ki Cheoun) is supported by the National Research Foundation of Korea (NRF-2014R1A2A2A05003548).

\section{References}

1. WMAP Collab. (D. N. Spergel et al.), Astrophys. J. Suppl. 148 (2003) 175. 
2. D. N. Spergel et al., Astrophys. J. Suppl. 170 (2007) 377.

3. J. Dunkley et al., Astrophys. J. Suppl. 180 (2009) 306.

4. D. Larson et al., Astrophys. J. Suppl. 192 (2011) 16.

5. C. L. Bennett et al., Astrophys. J. Suppl. 208 (2013) 20.

6. L. Sbordone et al., Astron. Astrophys. 522 (2010) A26.

7. B. D. Fields, Annu. Rev. Nucl. Part. Sci. 61 (2011) 47, arXiv:1203.3551 [astro-ph.CO].

8. P. Sikivie and Q. Yang, Phys. Rev. Lett. 103 (2009) 111301.

9. O. Erken, P. Sikivie, H. Tam and Q. Yang, Phys. Rev. D 85 (2012) 063520.

10. J. Jaeckel, J. Redondo and A. Ringwald, Phys. Rev. Lett. 101 (2008) 131801, arXiv:0804.4157 [astro-ph].

11. M. Kusakabe, A. B. Balantekin, T. Kajino and Y. Pehlivan, Phys. Lett. B 718 (2013) 704.

12. J. R. Ellis, K. A. Olive and E. Vangioni, Phys. Lett. B 619 (2005) 30, arXiv:astro$\mathrm{ph} / 0503023$.

13. M. Kusakabe, T. Kajino and G. J. Mathews, Phys. Rev. D 74 (2006) 023526, arXiv:astro-ph/0605255.

14. D. Lindley, Mon. Not. R. Astron. Soc. 188 (1979) 15p.

15. J. R. Ellis, D. V. Nanopoulos and S. Sarkar, Nucl. Phys. B 259 (1985) 175.

16. S. Dimopoulos, R. Esmailzadeh, L. J. Hall and G. D. Starkman, Astrophys. J. 330 (1988) 545.

17. M. H. Reno and D. Seckel, Phys. Rev. D 37 (1988) 3441.

18. N. Terasawa, M. Kawasaki and K. Sato, Nucl. Phys. B 302 (1988) 697.

19. M. Kawasaki and T. Moroi, Prog. Theor. Phys. 93 (1995) 879, arXiv:hep-ph/9403364.

20. M. Kawasaki and T. Moroi, Astrophys. J. 452 (1995) 506, arXiv:astro-ph/9412055.

21. K. Jedamzik, Phys. Rev. Lett. 84 (2000) 3248, arXiv:astro-ph/9909445.

22. M. Kawasaki, K. Kohri and T. Moroi, Phys. Rev. D 63 (2001) 103502, arXiv:hep$\mathrm{ph} / 0012279$.

23. R. H. Cyburt, J. R. Ellis, B. D. Fields and K. A. Olive, Phys. Rev. D 67 (2003) 103521, arXiv:astro-ph/0211258.

24. M. Kawasaki, K. Kohri and T. Moroi, Phys. Rev. D 71 (2005) 083502, arXiv:astro$\mathrm{ph} / 0408426$.

25. K. Jedamzik, Phys. Rev. D 70 (2004) 063524, arXiv:astro-ph/0402344.

26. K. Jedamzik, Phys. Rev. D 70 (2004) 083510, arXiv:astro-ph/0405583.

27. K. Jedamzik, Phys. Rev. D 74 (2006) 103509 arXiv:hep-ph/0604251.

28. M. Kusakabe et al., Phys. Rev. D 79 (2009) 123513, arXiv:0806.4040 [astro-ph].

29. R. H. Cyburt et al., J. Cosmol. Astropart. Part. 1010 (2010) 032, arXiv:1007.4173 [astro-ph.CO].

30. M. Pospelov and J. Pradler, Phys. Rev. D 82 (2010) 103514, arXiv:1006.4172 [hep-ph].

31. M. Kawasaki and M. Kusakabe, Phys. Rev. D 86 (2012) 063003.

32. D. G. Yamazaki and M. Kusakabe, Phys. Rev. D 86 (2012) 123006.

33. D. G. Yamazaki, M. Kusakabe, T. Kajino, G. J. Mathews and M.-K. Cheoun, Phys. Rev. D 90 (2014) 023001.

34. L. H. Kawano, NASA STI/Recon Technical Report No. 92, 25163 (1992); https://ntrs.nasa.gov/search.jsp?R=19920015920.

35. M. S. Smith, L. H. Kawano and R. A. Malaney, Astrophys. J. Suppl. 85 (1993) 219.

36. R. H. Cyburt et al., Astrophys. J. Suppl. 189 (2010) 240.

37. A. P. Serebrov and A. K. Fomin, Phys. Rev. C 82 (2010) 035501.

38. T. Shima et al., Phys. Rev. C 72 (2005) 044004, arXiv:nucl-ex/0509017.

39. T. Kii, T. Shima, Y. Nagai and T. Baba, Nucl. Instrum. Methods Phys. Res. A 552 (2005) 329. 
40. J. L. Feng, A. Rajaraman and F. Takayama, Phys. Rev. Lett. 91 (2003) 011302, arXiv:hep-ph/0302215.

41. J. L. Feng, A. Rajaraman and F. Takayama, Phys. Rev. D 68 (2003) 063504, arXiv:hep-ph/0306024.

42. D. G. Yamazaki, K. Ichiki, T. Kajino and G. J. Mathews, Phys. Rev. D 77 (2008) 043005.

43. D. G. Yamazaki, K. Ichiki and K. Takahashi, Phys. Rev. D 84 (2011) 123006.

44. D. G. Yamazaki, Phys. Rev. D 89 (2014) 083528.

45. D. G. Yamazaki, K. Ichiki, K. Umezu and H. Hanayama, Phys. Rev. D 74 (2006) 123518.

46. D. G. Yamazaki, Phys. Rev. D 93 (2016) 043004.

47. D. G. Yamazaki, K. Ichiki and T. Kajino, Astrophys. J. 625 (2005) L1.

48. D. G. Yamazaki, K. Ichiki, T. Kajino and G. J. Mathews, Astrophys. J. 646 (2006) 719 .

49. D. G. Yamazaki, K. Ichiki, T. Kajino and G. J. Mathews, Phys. Rev. D 78 (2008) 123001.

50. D. G. Yamazaki, K. Ichiki, T. Kajino and G. J. Mathews, Phys. Rev. D 81 (2010) 023008 .

51. D. G. Yamazaki, K. Ichiki, T. Kajino and G. J. Mathews, Phys. Rev. D 81 (2010) 103519.

52. D. G. Yamazaki, K. Ichiki, T. Kajino and G. J. Mathews, Adv. Astron. 2010 (2010) 586 .

53. D. G. Yamazaki, T. Kajino, G. J. Mathews and K. Ichiki, Phys. Rep. 517 (2012) 141.

54. D. G. Yamazaki, K. Ichiki and K. Takahashi, Phys. Rev. D 88 (2013) 103011.

55. R. Keisler et al., Astrophys. J. 743 (2011) 28.

56. C. L. Reichardt et al., Astrophys. J. 755 (2012) 70.

57. J. W. Fowler et al., Astrophys. J. 722 (2010) 1148.

58. S. Das et al., Astrophys. J. 729 (2011) 62.

59. J. Guy et al., Astron. Astrophys. 523 (2010) A7.

60. A. Conley et al., Astrophys. J. Suppl. 192 (2011) 1.

61. M. Sullivan et al., Astrophys. J. 737 (2011) 102.

62. O. Erken, P. Sikivie, H. Tam and Q. Yang, Phys. Rev. Lett. 108 (2012) 061304, arXiv:1104.4507 [astro-ph.CO].

63. E. Aver, K. A. Olive and E. D. Skillman, J. Cosmol. Astropart. Phys. 1005 (2010) 003, arXiv:1001.5218 [astro-ph.CO].

64. K. Lind, J. Melendez, M. Asplund, R. Collet and Z. Magic, Astron. Astrophys. 554 (2013) A96, arXiv:1003-4510 [astro-ph.GA]. 